\title{
Iterative Calibration of VISSIM Simulator Based on Genetic Algorithm
}

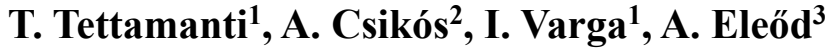 \\ ${ }^{1}$ Budapest University of Technology and Economics, Department of Control for \\ Transportation and Vehicle Systems, \\ Stoczek utca 2., 1111, Budapest, Hungary \\ Phone: +36 14632255 \\ e-mail: tettamanti@mail.bme.hu \\ ${ }^{2}$ Systems and Control Laboratory, Research Institute for Computer Sciences and \\ Automation, Hungarian Academy of Sciences \\ Kende utca 13-17, 1111, Budapest, Hungary \\ ${ }^{3}$ Budapest University of Technology and Economics, Department of Vehicle \\ Elements and Vehicle-Structure Analysis \\ Stoczek utca 2., 1111, Budapest, Hungary
}

Abstract: In the recent decade, computer simulation has been developed enormously in all engineering fields and has become a basic step of design. Accordingly, simulation plays a fundamental role in road traffic engineering offering a basis for the effective analysis of intelligent traffic control systems. A reliable simulator is able to provide effective analysis of a given traffic network if the applied simulation scenarios properly converge to the real-word situation. This requirement can be achieved based on the mixed use of prior real-world traffic measurements and proper simulation settings. The latter one, however, is not straightforward. Accordingly, the paper proposes a potential calibration method to create true-to-life VISSIM simulations. VISSIM is one of the most popular microscopic traffic simulator, a well-known software applied in engineering and scientific practice as well. Basically, a calibration technique with genetic algorithm optimization is proposed to reproduce realistic traffic on a network based on floating car speed data.

Keywords: Vissim simulator, calibration, genetic algorithm

\section{Introduction}

The continuous maintenance and development of road traffic control systems is indispensable due to the increasing traffic demands. Appropriate simulation represents the preceding stage of such engineering tasks. Moreover, the concept of intelligent transportation systems (ITS) of our days also requires the advanced use of informatics. 
As a consequence, when traffic engineers design intelligent solutions, the use of traffic simulators and mathematical optimization software is more and more expected during the development process and the validation phase as well. However, the proper use of simulators is important in order to avoid false simulation results. Namely, beside the several advantages of simulation, simulators also contain the danger of providing erroneous results in case of inappropriate simulation settings in the scenarios. Accordingly, calibration of simulation parameters is expected. Numerous researches have been conducted in this field providing efficient methods to optimize the most important settings, such as travel times, driving behavior parameters, saturation flow rates, etc. These parameters are mostly microscopic variables which can be successfully tuned based on the research results, e.g. [1][2][3].

In this paper, however, we focus only on the calibration of a macroscopic variable, the traffic demand which is one of the most important network parameter in the microscopic simulation. Moreover, traffic input is calibrated based on real-world floating car data (FCD) participating as sensors in the road network. FCD is typically collected from fleet cars equipped with a GPS receiver providing accurate speed data and GPS position logs. Beside fleet cars, cellular phone based methods (server and client side as well) are also available to collect individual speed data of travelers [4]. Another emerging technology is represented by the Bluetooth-based vehicle detection [5], which is already applied in few cities.

Apparently, the plethora of the novel measurement technologies offers the possibility to gather link average traffic speed for large networks. A well-known example is the Traffic functionality of Google Map. The sources of Google data are FCD information of fleet management companies and mobile data of private smartphone users. This service determines speed categories by using color codes displayed on links (see Figure $1)$.

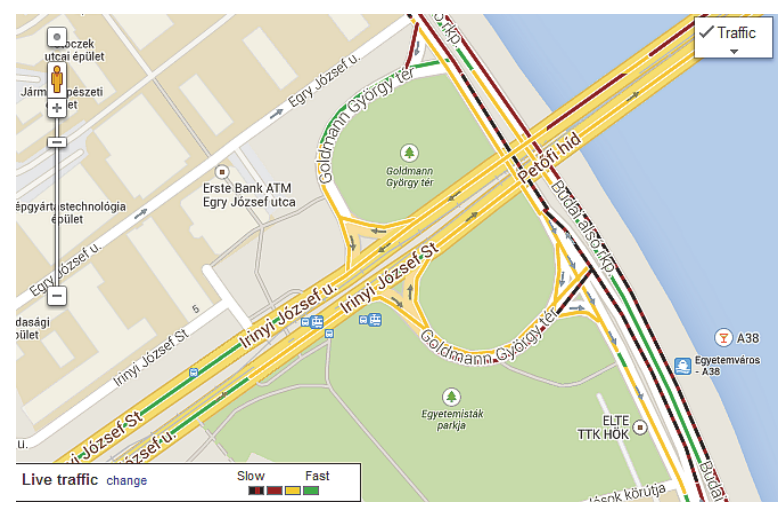

Figure 1. Google Map screenshot in Budapest

As demonstrated above, the traffic speed on road links is already available. Therefore, the problem is simply given: how to create a reliable traffic model based on FCD speed information and without any traffic flow measurement? The solution is not straightforward as the traffic demand (traffic input flows) must be determined only from 
speed data. Note, that a given speed can be induced by different traffic inputs. Moreover, in case of a bigger traffic network, a combination of several demand flows has to be taken into account. Practically, this is a reverse engineering problem which analytical solution is not possible as it is highly underdetermined. As a result of these reasons, an online and iterative optimization is proposed to be applied for the problem.

The paper is organized as follows. First, the applied microscopic traffic simulation environment is introduced. Then, the proposed iterative calibration method is described. Finally summary and evaluation is provided.

\section{Microscopic road traffic simulation with VISSIM}

Road traffic simulations can be realized on macroscopic and microscopic level. On the former level, traffic is described as a continuum by macroscopic parameters (e.g. traffic flow, traffic density, average travel times, etc). The latter modeling level provides a high-resolution description of traffic via dynamic modeling of each vehicle. Macroscopic modeling level clearly has the advantage in terms of computational demand, therefore it is mainly used on a large spatial and temporal domain while microscopic modeling comes into prominence when analyzing the traffic of small networks, covering a few intersections. On both levels, the proper knowledge of basic parameters the process is essential in the design of a valid traffic simulation, i.e. traffic demands and origin-destination data of the network. However, these data are not fully available in most cases. Therefore, intelligent calibration of such parameters is suggested (see next section).

Users may choose among many different road traffic simulators. The greater part of them is commercial software. Additionally, open source simulators are also available, developed by universities or research institutes. Each of them has advantages or drawbacks depending on the individual demands of the user. In our paper, the applied simulation environment is designated to be designed by using VISSIM [6]. Furthermore, the integrated VISSIM-MATLAB environment is applied as described in [7] as a state-of-the-art technology for advanced simulation.

VISSIM is a microscopic simulator based on the individual behavior of vehicles. The goal of the microscopic modeling approach is the accurate description of traffic dynamics. Thus, the simulated traffic network may be analyzed in detail. The simulator uses the so-called psycho-physical driver behavior model developed originally by Wiedemann [8]. VISSIM is widely used for diverse problems by traffic engineers in practice as well as by researchers for developments related to road traffic. VISSIM offers a user friendly graphical interface (GUI) through of which one can design the geometry of any type of road networks and set up simulations in a simple way. However, for several problems the GUI is not satisfying. This is the case, for example, when the user aims to access and manipulate VISSIM objects during the simulation dynamically. For this end, an additional interface [9] is offered based on the COM (Component Object Model) which is a technology to enable interprocess communication between software [10]. Via the VISSIM COM the user is able to manipulate the attributes of most of the internal objects dynamically. The first step of a $\mathrm{COM}$ based simulation is to create the COM client. Then, one can realize the parts or even the whole process of the simulation. 


\section{Genetic algorithm based calibration}

\subsection{The basic problem and assumptions}

The basic task is to reveal traffic demand, i.e. vehicle input flows entering the network. To achieve, this FCD based average speeds of road links can be applied where a link is determined as a road stretch between two signalized intersections. Moreover, measurement data of average turning rates of junctions need to be available. The microscopic parameters such as driving behavior, lane change are assumed to be well calibrated based on any existing methods cited in the introduction part for example. Real-world signal settings (green time split, offset, cycle time) are known and used in the simulation. The whole problem is depicted in Figure 2.

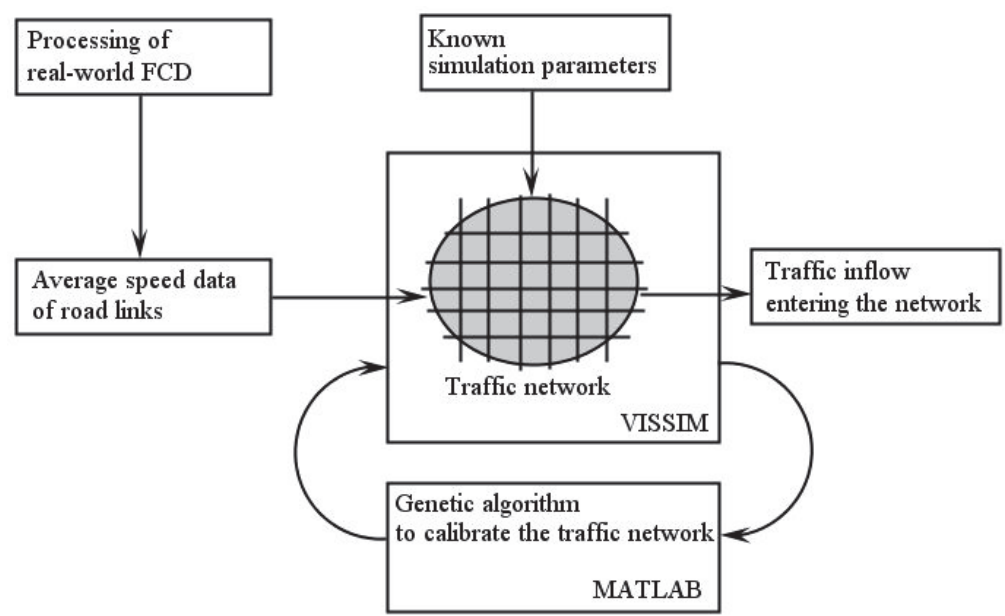

Figure 2. The basic calibration problem

\subsection{Iterative method for calibration}

The traditional method to optimization applies the derivative-based approach. However, if the objective function does not have a derivative, this approach cannot be used. In our case, the objective function contains a non-derivative term, i.e. the results of the VISSIM simulation run. A potential solution is to formalize and solve the optimization problem as a genetic algorithm (GA) problem.

The idea of using soft computing [11] for simulator calibration has already been recognized. However, the approach has only been used for the tuning of model parameters. In [3] a GA is used to optimize vehicle performance models in traffic simulators. In [12] the parameters of the car following and lane-changing dynamics of VISSIM are tuned using the particle swarm optimization method. The traffic simulator PARAMICS is calibrated in [13] by using a GA approach. [14] investigates a method for parameter optimization using a GA approach as well, tested in CORSIM and VISSIM simulators. 
In our contribution, an iterative method is proposed. By applying the Snapshot option of the simulator, the algorithm tries to fit the average link speeds to the traffic inflows (Vehicle Inputs in VISSIM). Basically, during the calibration process a discrete time window is iteratively simulated. The time window only moves on if the optimization criteria is fulfilled, i.e. the average speeds measured in the simulator do not differ from the real-world FCD speeds. The applied fitness function of the optimization is as follows:

$$
J(k)=\sum_{i=1}^{n}\left\|\frac{\bar{V}_{i}^{F C D}(k)-\bar{V}_{i}^{\text {VISSIM }}(Q(k))}{\bar{V}_{i}^{F C D}(k)}\right\|_{\infty} \rightarrow \min
$$

$\bar{V}_{i}^{F C D}(k)$ is the average speed of link $i$ originated from FCD information at time step $k$. $\bar{V}_{i}^{V I S S I M}(Q(k))$ denotes the average speed of link $i$ produced by VISSIM in the previous simulation time window $k . Q(k)$ represents the applied vehicle input parameter.

Practically, the fitness function penalizes the relative deviation between simulated and real-world speed of each road link. The function is expressed as the infinity norm of the vector of traffic speeds. The GA based optimization runs within each time window until the termination criterion is achieved, i.e. $J(k)<\varepsilon$. For example, if termination parameter is set to $\varepsilon=0.2$, the maximal admissible relative deviation of the average speed is $20 \%$.

The complete calibration method is given by Figure 3. The calibration is realized in VISSIM-MATLAB environment by using COM programming, see [6]. 


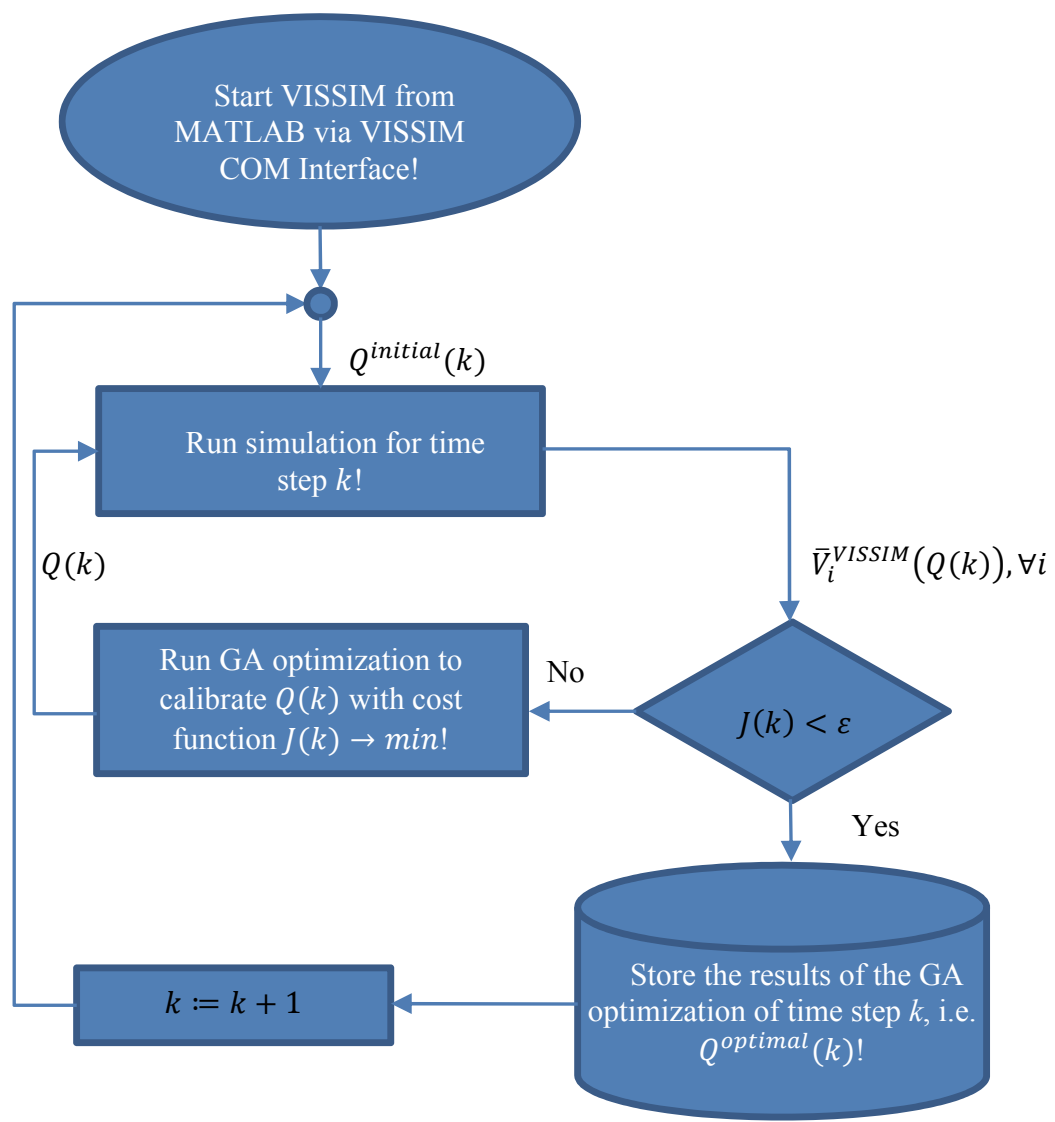

Figure 3. Workflow of the iterative calibration of VISSIM simulator

\subsection{Example for the calibration}

To demonstrate the proposed calibration technique, a simple example was investigated. Based on average speed functions deduced from real-world FCD data (iData Ltd.), a test link (Budapest, at Oktogon square) was calibrated for a peak period between 7:00 and 09:30. The calibration method has been applied with 1 minute sample time and termination parameter $\varepsilon=0.2$.

The variation of the fitness function $J(k)$ can be observed in Fig. 4. This reflects the maximum relative error obtained during the calibration process, i.e. the difference between the link speed based achieved from the calibrated parameters and the realworld mean speed. Practically, $J(k)$ always remains under $22 \%$, which can be considered a satisfying accuracy for calibration. 


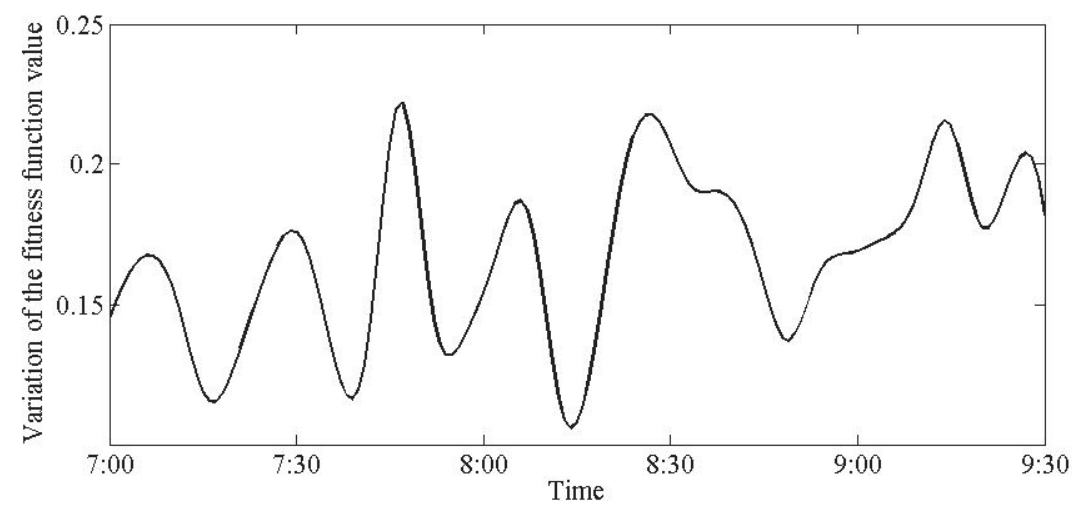

Figure 4. Fitness function value during the calibration

\section{Summary}

In our work, a genetic algorithm based calibration method is suggested for traffic simulators, reproducing realistic traffic conditions on a network represented by FCD data. The tuned variables of the simulation are the traffic flow inputs of the network. The obtained network link speed values should fit to the FCD speed data.

By using real-world floating car traffic data and a microscopic traffic simulator, the proposed calibration algorithm is efficiently applied and tested under different traffic conditions.

The proposed method was demonstrated by using the well-known VISSIM simulator. Nevertheless, it is emphasized that the technique can be applied for any type of microscopic traffic simulators.

\section{Acknowledgement}

This work is connected to the scientific program of EITKIC-12-1-2012-0001 project (supported by the Hungarian Government, managed by the National Development Agency, financed by the Research and Technology Innovation Fund), and TÁMOP4.2.2.C-11/1/KONV-2012-0012: Smarter Transport project (supported by the Hungarian Government, co-financed by the European Social Fund). The FCD measurement data set, used for the simulation test, has been provided by iData Ltd., which is gratefully acknowledged.

\section{References}

[1] Columbia River Crossing, VISSIM calibration and validation. Technical report, 2006.

[2] Park B, Schneeberger JD: Microscopic Simulation Model Calibration and Validation, Case Study of VISSIM Simulation Model for a Coordinated Actuated Signal System. Transportation Research Record: Journal of the Transportation Research Board, Vol. 1856, pp. 185-192, 2003.

DOI: $\underline{10.3141 / 1856-20}$ 
[3] Cunha AL, Bessa JE Jr, Setti JR: Genetic Algorithm for the Calibration of Vehicle Performance Models of Microscopic Traffic Simulators. Progress in Artificial Intelligence, Lecture Notes in Computer Science, Vol. 5816, pp. 3$14,2009$.

DOI: $10.1007 / 978-3-642-04686-5 \quad 1$

[4] T. Tettamanti, I. Varga, Mobile phone location area based traffic flow estimation in urban road traffic. Columbia International Publishing, Advances in Civil and Environmental Engineering, 1(1):1-15, 2014.

[5] Qing O: Fusing Heterogeneous Traffic Data: Parsimonious Approaches using Data-Data Consistency. PhD Thesis, Delft University of Technology, 2011.

[6] Tettamanti T, Varga I: Development of road traffic control by using integrated VISSIM-MATLAB simulation environment. Periodica Polytechnica - Civil Engineering. Vol. 56(1), pp. 43-49, 2012.

DOI: $10.3311 /$ pp.ci.2012-1.05

[7] PTV, VISSIM 5.4 User Manual. PTV Planung Transport Verkehr AG, Germany, 2012.

[8] Wiedemann R, Simulation des Straßenverkehrsflusses. Schriftenreihe des Instituts für Verkehrswesen der Universität Karlsruhe, 8, 1974.

[9] PTV, VISSIM - COM Interface Manual 5.4, PTV Planung Transport Verkehr AG, Germany, 2012.

[10]Box D: Essential COM. Addison-Wesley, ISBN 0-201-63446-5, 1998.

[11] Weise T: Global Optimization Algorithms - Theory and Application, 2008

[12] Aghabayk K, Sarvi M, Young W, Kautzsch L: A novel methodology for evolutionary calibration of Vissim by multi-threading. Australasian Transport Research Forum 2013 Proceedings, Australia, 2013

[13] Chu L, Liu HX, Oh JS, Recker W: A calibration procedure for microscopic traffic simulation. Proceedings of the 2003 Intelligent Transportation Systems. Vol.2, pp. $1574-1579,2003$.

DOI: $10.1109 /$ ITSC.2003.1252749

[14] Park B, Won J, Yun I: Application of Microscopic Simulation Model Calibration and Validation Procedure: A Case Study of Coordinated Actuated Signal System. Transportation Research Record, Vol. 1978, pp. 113-133, 2006. DOI: $\underline{10.3141 / 1978-16}$ 